\title{
Modelo de demanda de dinero para firmas: aspectos teóricos, metodología y resultados
}

\author{
Money Demand Model for Firms: \\ Theoretical Aspects, Methodology and Results \\ Modelo de demanda de dinheiro para firmas: \\ aspectos teóricos, metodologia e resultados
}

\author{
Wilson Vera Lasso \\ Universidad Complutense de Madrid. Madrid, España \\ wvera@hotmail.com \\ https://orcid.org/0000-0001-5323-3519 \\ Andrés Dillon Ávila \\ Facultad Latinoamericana de Ciencias Sociales. Quito, Ecuador \\ andresdillon29@hotmail.com \\ https://orcid.org/0000-0003-2819-2668 \\ Ricardo Erazo Loor \\ University of Nottingham. Nottingham, Inglaterra \\ rjerazol88@gmail.com \\ https://orcid.org/0000-0002-9935-0355
}

DOI: https://doi.org/10.32719/25506641.2021.9.6

Recibido: 20 de mayo de 2020 • Revisado: 26 de junio de 2020 • Aceptado: 31 de julio de 2020

Artículo de investigación

Licencia Creative Commons

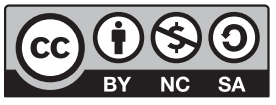




\section{Resumen}

El propósito de este artículo es contribuir con los elementos teóricos, metodológicos y empíricos de la demanda de dinero para una empresa, para que la gestión de la tesorería de firmas y de instituciones financieras que utilizan un flujo importante de recursos monetarios líquidos permita, por un lado, minimizar los costos de oportunidad de mantener un exceso de dinero en efectivo y, por otro lado, cubrir oportunamente con sus obligaciones de corto plazo. Para esto se plantea, en una empresa mediana de comercialización de software, la aplicación del modelo MOV (Miller, Orr y Vera) de demanda de dinero para firmas, cuyo sustento teórico es el planteamiento inicial de Miller y Orr (1968), además de la contribución de Vera (2004). A partir de los resultados obtenidos en esta investigación, se concluye que es posible el manejo eficiente de la liquidez, minimizando los costos de transacción y maximizando la rentabilidad de las inversiones en títulos valores.

Palabras clave: demanda de dinero, tesorería, economía de empresa, política de inversión.

JEL: M2 Economía de la empresa.

\section{Abstract}

The purpose of this article is to contribute with the theoretical, methodological and empirical elements of the money demand for a company, so that the management of the treasury of firms and financial institutions using a significant flow of liquid monetary resources allows, on one hand, to minimize the opportunity costs of maintaining excess cash and, and on the other hand, to cover their short-term obligations in a timely manner. For this, in a medium-sized software marketing company, the application of the MOV model (Miller, Orr and Vera) of demand for money for firms is proposed, whose theoretical support is the initial approach of Miller and Orr, 1968, in addition to the contribution of Vera, 2004.

From the obtained results in this research, it is concluded that efficient liquidity management is possible minimizing transaction costs and maximizing the investment profitability in securities.

Keywords: Money demand, treasury, business economics, investment policy.

JEL: M2 Economics of the company.

\section{Resumo}

O propósito deste artigo é contribuir com os elementos teóricos, metodológicos e empíricos da demanda por dinheiro para uma empresa para que a gestão de tesouraria de firmas e de instituições financeiras que utilizam um fluxo importante de recursos monetários líquidos permita, por um lado, minimizar os custos de oportunidade de manter um excesso de dinheiro em caixa e, por outro, cobrir oportunamente com suas obrigações a curto prazo. Para tanto, propõe-se, em uma empresa mediana de comercialização de software, a aplicação do modelo MOV (Miller, Orr e Vera) de demanda por dinheiro para firmas, cujo alicerce teórico é a proposta inicial de Miller e Orr, 1968, e a contribuição de Vera, 
2004. A partir dos resultados obtidos nesta pesquisa, conclui-se que é possível a gestão eficiente da liquidez, minimizando-se os custos de transação e maximizando a rentabilidade dos investimentos em títulos de crédito.

Palavras-chave: Demanda por dinheiro, tesouraria, economia empresarial, política de investimento.

JEL: M2 Economia empresarial.

\section{Introducción}

U no de los temas que más preocupa a los investigadores de la economía es el funcionamiento del mercado de dinero y dentro de esto, la dinámica de la oferta y la demanda de dinero. Los planteamientos teóricos y los modelos econométricos desarrollados a partir de esta temática han permitido a los hacedores de la política pública tomar decisiones en el ámbito monetario, con el propósito de influir sobre la economía real. Una apropiada modelización, en este caso, de los temas monetarios, permite determinar los mecanismos más óptimos de transmisión de la política monetaria y, por tanto, de la efectividad de la política pública (Pigou 1917; Goldman 1974; Guissarri 1986; Tobin 1956; Keynes 1936; Fisher 1986).

Dentro de este contexto, entender el porqué los agentes económicos, no solo los individuos sino las empresas, desean un determinado nivel de saldo de activos financieros líquidos, ha llevado a los estudiosos en la materia a generar una abundante literatura sobre la demanda de dinero. A este respecto, se han desarrollado estudios desde distintos enfoques, por ejemplo, la consideración del dinero como un bien de consumo, el tratamiento del dinero como si fuese un factor productivo, la incorporación de los saldos líquidos en la función de utilidad, la identificación del dinero como un activo financiero especialmente en el marco de modelos de selección de cartera, entre otros (Miller y Orr 1968; Feige y Parkin 1971; Baumol 1952; Milbourne 1983; Tapiero y Zuckerman 1980).

Sin embargo, dado el rol fundamental que cumple el dinero como medio de pago, la mayoría de las investigaciones se refieren a la demanda de dinero por motivo de transacción y no como precaución o especulación; es decir, se considera que los bienes de la economía solo pueden ser adquiridos a cambio de dinero, justificándose la demanda de dinero que realizan las personas y 
las empresas por los desfases temporales entre las corrientes de ingresos y pagos, unidos al hecho de la existencia de costes de transacción, es decir de costes asociados a la conversión del resto de activos en dinero (Aznar y Pozo 1998).

Desde esta perspectiva, se han formulado varias investigaciones con modelos en los que los agentes económicos no se enfrentan a incertidumbre cuando toman sus decisiones, como los casos de las aportaciones que desarrollan en algún aspecto los conocidos modelos de inventario de Baumol (1952) y Tobin (1956), el trabajo de Feige y Parkin (1971) que amplían el modelo de Tobin, permitiendo la acumulación de bienes, la investigación de Santomero (1974), en el que se formula una versión con dos tipos de dinero, los estudios de Jovanovic (1982) y Romer (1987) que permiten que el período entre transacciones sea endógeno, entre otros. Una segunda línea de trabajos es aquella que considera la existencia de incertidumbre en la toma de decisiones por parte de los agentes; así, podemos citar, entre otros: la investigación de Miller y Orr (1968), en el cual los agentes tratan de mantener sus tenencias de saldos líquidos dentro de un intervalo óptimo sin conocer con exactitud el volumen de cobros y pagos del período; por su parte, el análisis de Goldman (1974) formula un modelo en el que existen dos períodos de decisión y en el que los individuos deben determinar su demanda de activos antes de conocer la cuantía de sus gastos, los cuales dependen del estado del entorno en que se encuentren. El artículo de Milbourne (1983) establece un modelo en el cual los saldos líquidos en manos de los agentes se ven afectados por shocks estocásticos (Aznar y Pozo 1998).

En este estudio se analizan los aspectos teóricos, metodológicos y los resultados de la demanda de dinero para una empresa, a partir del modelo planteado por (Miller y Orr 1968). En este sentido se abordan, después de la introducción, los aspectos teóricos en que se sustenta el modelo; en tercer lugar, se analizan los puntos metodológicos del modelo, para luego continuar con un cuarto punto donde se realiza una aplicación del modelo para el caso de una determinada firma y finalmente se presentan las conclusiones. 


\section{Marco teórico}

Una cuestión relevante que se plantea en las empresas se refiere a cuál debe ser el nivel óptimo de tesorería que debe mantener para hacer frente a sus pagos diarios, sin que ello signifique costos de oportunidad de mantener liquidez en opciones que le generen ciertos niveles de rentabilidad. Como bien lo señala Mies y Soto (2000), los modelos basados en la teoría cuantitativa del dinero no consideran como fundamento la idea de que los agentes económicos deban fijar un monto óptimo de saldos monetarios. Una primera aproximación desde este enfoque aparece con el modelo keynesiano, que cuenta con una noción implícita del proceso optimizador; pero su modelación no fue la más adecuada, ante esta necesidad no cubierta surgen los modelos de inventarios. ${ }^{1}$ En este contexto, Baumol (1952) y Tobin (1956) desarrollaron formalmente los primeros modelos de optimización para la demanda de dinero por transacciones, utilizando como base los modelos de inventarios. Estos autores plantean una optimización al minimizar la pérdida de intereses y los costos de transacción respecto de la transformación de bonos a dinero en cada operación.

Los principales supuestos asumidos por Baumol (1952) en su modelo son: i) las necesidades de efectivo de la empresa son conocidos con certeza; ii) el uso de efectivo de la empresa tiene lugar de manera uniforme en un período que se conoce con seguridad; iii) el costo de oportunidad de mantener dinero en efectivo es constante y de conocimiento de la empresa; y, iv) el costo de transacción por convertir títulos a efectivo es constante y conocido por la empresa. El alto nivel de certidumbre de la información presente en este modelo implica que su aplicación pueda no ser, necesariamente, efectiva para las empresas e instituciones, de ahí la relevancia del modelo propuesto por Miller y Orr (1968).

1. Es relevante considerar que los autores plantean que "Una limitación importante de los modelos de inventarios es que empíricamente estos solo explican una pequeña fracción de las tenencias de dinero por transacciones que se mantienen normalmente en una economía. Además, presentan la falencia de tratar el flujo de ingresos y de costos exógenamente, impidiendo la generalización a un contexto dinámico. No obstante, estos modelos explicitan directamente el tipo de servicio que provee el dinero, característica que no poseen otros modelos más complejos". (Mies y Soto 2000, 9) 
Adicionalmente, Tobin (1958) desarrolla otro importante aporte al construir un modelo en que los saldos monetarios dependen de una cartera de activos bajo condiciones de incertidumbre. Es decir, que la asignación de recursos que realicen los agentes económicos, dado su grado de aversión al riesgo y nivel de riqueza, variarán dependiendo si las realizan entre un activo libre de riesgo (el dinero) o un activo riesgoso (bonos), con la condición de que el retorno esperado supere al del dinero. ${ }^{2}$

En esta línea, haremos un análisis más exhaustivo al desarrollo metodológico que realizaron Miller y Orr (1968), quienes plantearon una serie de técnicas para la toma de decisiones relacionadas a la gestión de la tesorería de una empresa, con el objetivo de alcanzar un saldo óptimo de liquidez según las necesidades de cada organización. Este planteamiento teórico establece que en una compañía existen una serie de flujos diarios de entrada y salida de efectivo, los cuales no son constantes, es decir, son aleatorios; al igual que acontece con los individuos, en las compañías tanto los ingresos como los gastos no son regulares y dependen de la situación comercial y de ventas que se producen en la gestión diaria. Lo significativo es que, mediante la aplicación del modelo, una firma puede ir evaluando y gestionado permanentemente el manejo de la tesorería, tanto en etapas de exceso de liquidez como en situación de escasez de dinero líquido, con lo cual puede mantener con relativa normalidad su actividad económica. Por tanto, la imprevisibilidad de los flujos de recursos monetarios afecta a la toma de decisiones de inversión o desinversión de títulos que la empresa registra entre sus activos, lo cual facilita tener un nivel de liquidez equilibrado (Miller y Orr 1968).

En este contexto, el modelo de demanda por dinero de estos autores indica como las organizaciones deberían gestionar los saldos de tesorería, en caso de que no se pueda predecir diariamente las entradas y salidas. La regla es permitir que varíe libremente el saldo de tesorería hasta que alcance un límite superior o inferior. Cuando esto sucede, la empresa compra o vende títulos para volver al saldo deseado. Se asume que el ingreso de los agen-

2. Por su parte, este modelo presenta algunas limitaciones puesto que el dinero no posee un retorno libre de riesgo en términos reales, concepto pertinente para el agente racional y, por otro lado, en muchas economías existen activos que poseen similares características de riesgo que el dinero, pero que entregan un mayor retorno, lo cual implicaría que bajo los mismos supuestos de este modelo, el dinero no sería demandado. 
tes sigue un proceso de camino aleatorio y que el problema del consumidor consiste en minimizar los costos de transacción y los intereses netos que se pierden al mantener dinero. En este caso, la mantención de saldos monetarios sigue una regla: los saldos de tesorería no pueden estar por encima de un determinado límite superior ni por debajo de un límite inferior, caso contario, las empresas deben ajustar su portafolio de manera que alcance un determinado nivel deseable de tesorería (Mies y Soto 2000).

Miller y Orr (1968) ampliaron el modelo de Baumol introduciendo un proceso de generación aleatoria para los cambios diarios en el saldo de efectivo. Esto significa que los cambios en el saldo de efectivo, a lo largo de un período, son aleatorios tanto en tamaño como en dirección, tendiendo a una distribución normal a medida que aumenta el número de períodos observados. Posteriormente, cuando el saldo sube durante un cierto tiempo y alcanza un punto determinado, el administrador financiero o el tesorero ordena una transferencia de una cantidad de efectivo a inversiones temporales, es decir, coloca una determinada cantidad de efectivo, por lo que el saldo de efectivo vuelve a un nivel más bajo. Por el contrario, cuando el nivel de efectivo durante algún período llega a un nivel muy bajo o cero, las inversiones son vencidas haciéndose una transferencia a la cuenta de efectivo de la empresa, para llegar a un nivel más alto del saldo de efectivo (Reyes 2012).

La utilización del proceso aleatorio para los cambios en el saldo de efectivo, adoptado por Miller y Orr, ha sido previamente cuestionada por autores como Tapiero y Zuckerman (1980), quienes establecen otra posible solución al manejo de efectivo cuando su ingreso y demanda se encuentran dadas por un proceso de distribución de Poisson en lugar de un proceso aleatorio (random walk) utilizado por Miller y Orr. Por su parte, Higson, Yoshikatsu, y Tippett (2010), denotan que el proceso aleatorio implicaría que la demanda de efectivo de la organización no crece en el tiempo, lo cual a su vez significaría que la empresa, al momento de crecer, no incrementaría su demanda de dinero. Es así como se establece un modelo de manejo de efectivo en el cual los movimientos de efectivo dependen del tamaño de la empresa en base a su producción, mostrando resultados distintos a los presentados por Miller y Orr, en los casos en que el flujo de efectivo presenta un proceso no estacionario. Esto demuestra que el modelo aún cuenta con espacio para mejorar; sin embargo, el uso final del modelo dependerá de la información con la que se 
cuente y el tipo de institución o empresa en la que se quiera aplicar el mismo.

Es importante definir los conceptos de efectivo y de valores negociables que se utilizan en este trabajo de investigación. El efectivo se refiere al dinero de curso legal que dispone la empresa en caja o en los saldos de las cuentas corrientes en los bancos, es decir, los recursos monetarios que no producen ninguna rentabilidad para la empresa; en tanto que, los valores negociables son aquellas inversiones temporales que realiza la empresa, utilizando el dinero improductivo y que su convertibilidad en efectivo se lo pueda hacer con cierta rapidez, aunque el valor a la fecha en que cambia no necesariamente sea el original, lo que significa que la empresa pueda obtener una pérdida o una ganancia (Higuerey 2007).

El propósito de la empresa, a través del responsable de la tesorería, es mantener niveles de saldo de efectivo adecuados, de manera que la posición en efectivo implique menores costos o que sea un mecanismo generador de utilidades a través de inversiones temporales en valores negociables. La decisión que las empresas o los individuos toman sobre la cantidad de dinero que desean mantener en un determinado período, depende fundamentalmente de sus ventajas e inconvenientes. La principal ventaja es tener dinero disponible para efectuar pagos de una manera inmediata, en tanto que el inconveniente se expresa a través del costo de oportunidad que se genera en términos de mantener ese dinero disponible y que puede ser el interés que se deja de percibir en activos más rentables en una economía con altos niveles de inflación, es la pérdida de poder adquisitivo.

En función de estas ventajas e inconvenientes, se distinguen tres importantes razones por las cuales el público desea demandar y conservar dinero en su poder: transacción, precaución y especulación. ${ }^{3}$ En el caso de las empresas, necesitan dinero para pagos a sus proveedores, para los salarios, entre otros. Esta necesidad de dinero surge porque los ingresos y los pagos de los agentes

3. El aporte al análisis de la demanda por dinero realizado por Keynes (1936) es determinar los tres motivos que inducen a los individuos a mantener saldos monetarios: transacción, precaución y especulación. El motivo de transacción se deriva de la necesidad que tienen los individuos de cubrir la brecha que se produce entre los ingresos generados y los gastos planeados. El motivo de precaución, en cambio, enfatiza el deseo de las personas de mantener dinero para hacer frente a gastos no planeados e inesperados. El motivo de especulación recoge el efecto de la incertidumbre acerca de la evolución de las variables macroeconómicas sobre las tenencias de dinero (Mies y Soto 2000). 
económicos no están perfectamente sincronizados. La demanda de dinero por transacción depende de la frecuencia con la que la gente obtenga ingresos. La pauta aquí es la siguiente: cuanto menos frecuentemente cobre una empresa por unidad de tiempo, mayor será la cantidad de dinero que deberá guardar para afrontar el pago de sus compras. Las empresas demandan dinero como precaución ante pagos inesperadamente elevados (por ejemplo, una avería de un camión) o ingresos inesperadamente bajos (por ejemplo, la posibilidad de pérdidas importantes en la empresa). Cuanto mayor sea la demanda por precaución, mayor es la protección o el colchón que posee una empresa contra el riesgo de quedarse sin dinero. Por otro lado, las empresas preferirán mantener parte de su riqueza en forma de dinero ante la expectativa de que se produzca un aumento del tipo de interés en otros activos financieros. Cuanto mayor sea el tipo de interés, menor cantidad de dinero se demandará pues se querrán comprar más activos financieros de alta rentabilidad (Ramos 2015).

Entre los motivos indicados, Guissarri (1986) analiza otro enfoque sobre la demanda de dinero, involucrando a la informalidad de los mercados como un determinante principal. En su estudio señala que, aspectos como los precios de los bienes sustitutos (depósitos a la vista), también inciden en la demanda de especies monetarias en circulación, considerando que una de las razones de demandar dinero es su utilización como un medio de pago de los agentes económicos; mientras que los depósitos a plazo son sustitutos perfectos de conservación de valor o atesoramiento de riqueza.

Además de los motivos por los cuales se demanda dinero, es importante mencionar los estudios de Fisher (1986) ${ }^{4}$ y Pigou (1917) desde la perspectiva de la teoría cuantitativa del dinero, son quienes resaltan que la demanda de dinero parte de optimizaciones lógicas (mayor utilidad, menores costos de transacción) de las empresas y hogares. Dichos enfoques fueron modificados y consolidados desde la visión keynesiana en la cual se determinó que la demanda de dinero estaría relacionada a la realización de transacciones, la precaución frente a eventos impredecibles y la especulación financiera. En esta misma línea, Mies y Soto (2000) evidencian la complejidad de relacionar el

4. Es importante indicar que, desde una perspectiva macroeconómica, el enfoque de Fisher no es una teorización de la demanda por dinero en sentido estricto, sino que corresponde a una reinterpretación causal de la identidad contable del gasto, que ya había adelantado Hume (1752) al reflexionar sobre las necesidades de dinero de una nación. 
valor de producción de un bien y su valor fiduciario representado a través del dinero, siendo su costo marginal cercano a cero; mientras que la convención social finalmente es quien determina su aceptación y confianza.

\section{Metodología}

El modelo analizado se basa en una función de costos que incluye el costo de realizar una transferencia hacia y desde el efectivo (costos fijos y variables de transacción) y el costo de oportunidad por mantener efectivo en caja. A los cambios en el saldo de efectivo se les permite ascender hasta alcanzar un nivel $\mathrm{H}$ (que se calcula) antes de decidir reducirlo hasta un nivel óptimo de caja llamado $\mathrm{Z}$, invirtiendo entonces la diferencia entre el monto al que ha llegado y Z. Al continuar las operaciones diarias, si se alcanza el punto mínimo (cero o un saldo mínimo de caja prefijado o nivel L), se hacen líquidos una parte de la inversión para llevar el efectivo otra vez al valor Z (ver gráfico 1).

\section{Gráfico 1}

\section{Muestra la representación del modelo de demanda de efectivo de Miller y Orr}

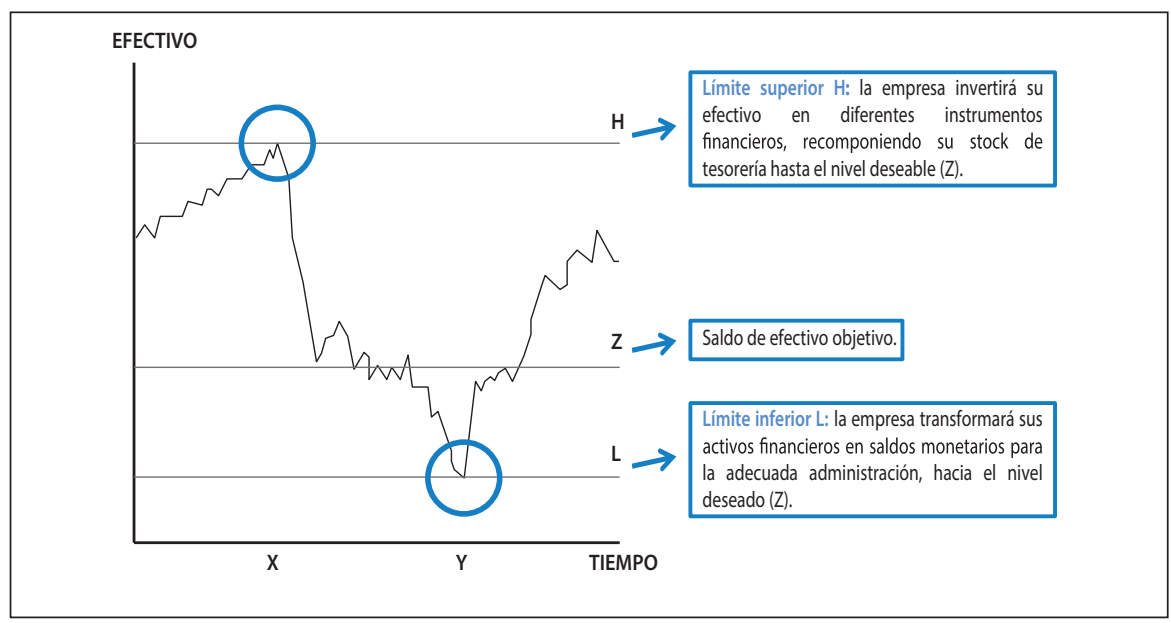

Fuente: Ross (1998). 
Entonces, el modelo calcula el límite superior $\mathrm{H}$ y el punto al cual debe devolverse el saldo de caja después de cada transferencia desde o hacia la cuenta de efectivo, o sea Z, de manera que se minimice la función de costo total de administración de efectivo.

El modelo evaluado por Miller y Orr (1968) analiza la óptima gestión de los saldos de tesorería de las empresas, con el objeto de anticipar las entradas y salidas, plantea un nivel deseable de saldo de tesorería y los límites superior e inferior para dicho stock y considera los siguientes aspectos:

- Costos de transacción (compra y venta de títulos negociables, F, es fijo).

- Costo de oportunidad porcentual por período de tenencia de efectivo, K, es la tasa de interés diaria de los títulos negociables.

- El nivel que minimiza los costos esperados de transacción y de oportunidad de los saldos de tesorería (banda Z-H).

- Existencias mínimas que garanticen la operación de dicha empresa (L). Para su aplicación se requiere de:

a) Fijar el límite inferior.

b) Calcular la desviación estándar de los flujos de caja diarios.

c) Determinar la tasa de interés.

d) Ponderar los costos de transacción de la compra y venta de los títulos negociables.

El modelo plantea que la empresa debe fijar un límite inferior sobre la base de la experiencia del tesorero de la organización, sin embargo, debido a que el límite inferior es establecido de forma $a d$ hoc, puede depender de cuánto nivel de riesgo de insuficiencia de efectivo está dispuesta la empresa a tolerar. Para solventar este problema Vera (2004) incluye en el modelo original de Miller y Orr un inventario de seguridad, como un elemento de protección contra la incertidumbre de la demanda, que toma en consideración el tiempo de entrega del suministro.

Para evitar problemas en el servicio al cliente y ahorrarse los costos ocultos de no contar con los componentes necesarios, las compañías mantienen un acopio de seguridad. Este inventario de seguridad es una protección contra la incertidumbre de la demanda, del tiempo de entrega y del suministro (Krajewski, Ritzman y Malhotra 2013).

El inventario de seguridad es: 


\section{$z \sigma \sqrt{d}$}

Donde:

$\mathrm{Z}=$ valor de la distribución normal.

$\sigma=$ desviación estándar de los datos.

$\mathrm{d}=$ días que demora la entrega.

Es decir, para la estimación del límite inferior se debe calcular el inventario de seguridad explicado anteriormente, por lo que el modelo con esta consideración se redefine como Modelo de Miller, Orr y Vera (MOV), de manera que al modelo original se incluye un método estadístico para estimar el inventario de seguridad, de manera que el mismo ya no se sustente en estimaciones subjetivas.

En resumen, para poder aplicar el modelo MOV para el análisis de la demanda de dinero para firmas, se requiere:

- Fijar el límite inferior, utilizando el cálculo del inventario de seguridad.

- Calcular la desviación estándar de los flujos de caja diarios, según el período analizado.

- Determinar la tasa de interés, representada por el rendimiento promedio ponderado de las inversiones en títulos valores que la empresa realice.

- Ponderar los costos que implican transformar dinero en efectivo a inversiones en títulos valores y viceversa.

Con lo cual la formulación matemática para la fijación de los límites superior e inferior y el nivel deseable es la siguiente:
Límite superior

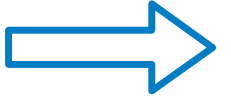$$
H^{*}=3 Z *-2 L
$$
Nivel deseable

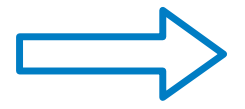$$
Z^{*}=\sqrt[3]{\frac{3 F \sigma^{2}}{4 K}}+L
$$
Límite inferior

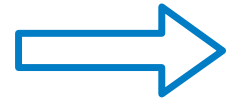

$$
L=z \sigma \sqrt{d}
$$


En la tabla 1 se muestra una explicación de cada componente de las fórmulas indicadas:

Tabla 1

\section{Resumen explicativo de las variables del modelo}

\begin{tabular}{|l|c|l|}
\hline \multicolumn{1}{|c|}{ Variable } & Símbolo & \multicolumn{1}{c|}{ Definición } \\
\hline Límite superior & $\mathrm{H}^{*}$ & $\begin{array}{l}\text { Monto superior de caja en el cual la empresa no debe } \\
\text { sobrepasar y deberá invertir en títulos negociables } \\
\text { para disminuir el saldo de liquidez. }\end{array}$ \\
\hline Nivel deseable & $\mathrm{Z}^{*}$ & $\begin{array}{l}\text { Saldo en que la empresa optimiza su nivel de liquidez, } \\
\text { en el cual no le genera costos de oportunidad ni } \\
\text { riesgos de liquidez. }\end{array}$ \\
\hline Límite inferior & $\mathrm{L}$ & $\begin{array}{l}\text { Monto inferior de caja en el cual la empresa no debe } \\
\text { reducir y deberá liquidar títulos negociables de manera } \\
\text { que pueda obtener un mayor nivel de liquidez. }\end{array}$ \\
\hline $\begin{array}{l}\text { Costo de transacción de compra } \\
\text { y venta de títulos valores }\end{array}$ & $\mathrm{F}$ & $\begin{array}{l}\text { Costos que debe asumir la empresa para comprar y } \\
\text { vender títulos negociables, que le permitirán reducir o } \\
\text { aumentar niveles de liquidez según sea el caso. }\end{array}$ \\
\hline $\begin{array}{l}\text { Varianza de los flujos de caja } \\
\text { netos diarios }\end{array}$ & $\sigma$ & $\begin{array}{l}\text { Es la medida de dispersión que representa la } \\
\text { variabilidad de una serie de datos respecto a su media. }\end{array}$ \\
\hline $\begin{array}{l}\text { Tasa de interés de los títulos } \\
\text { negociables }\end{array}$ & $\mathrm{K}$ & $\begin{array}{l}\text { Tasa de interés promedio de oportunidad si la empresa } \\
\text { decide invertir en otras actividades. }\end{array}$ \\
\hline Número de días & $\mathrm{d}$ & $\begin{array}{l}\text { Número de días que toma transformar el dinero líquido } \\
\text { en inversiones temporales y viceversa. }\end{array}$ \\
\hline
\end{tabular}

Fuente: adaptado de Ross (1998).

De esta manera, a diferencia de los estudios descritos, el modelo propuesto, a más de convertirse en una herramienta de aplicación empírica versátil para varias empresas, su conceptualización metodológica adapta la variabilidad (aleatoriedad) que podría observarse en los activos líquidos, especialmente en momentos en que su disponibilidad incide de forma directa en la capacidad de respuesta de la tesorería; optando en una alternativa cuantitativa a considerar respecto de las decisiones de inversión de la empresa, sin generar afectaciones sustanciales en los flujos de caja. 


\section{Resultados}

Una vez analizado el planteamiento teórico y metodológico del modelo MOV, se plantea a continuación una aplicación práctica para una empresa determinada. Para esto se utilizó el saldo diario de tesorería de una empresa cuyo principal giro de negocio es la venta de servicios de programación y software a compañías. Los límites fueron calculados en base a la información de la compañía entre enero de 2016 y diciembre de 2017.

El saldo promedio que la empresa presentó en su tesorería fue de USD 22.000. El costo de transacción de compra y venta de los títulos valores corresponde a USD 19,8 y la tasa de interés diaria de los títulos negociables corresponde al 0,003\%, en cuanto al tiempo de cambiar de posición entre efectivo y adquisición de títulos valores es de cuatro días.

De la serie histórica se obtiene que la desviación estándar es de 1,49 y la varianza, de 2,22, sobre la base de 499 observaciones. El valor máximo registrado fue de USD 42.600 y el saldo mínimo fue de USD 35.000 (ver tabla 2).

Tabla 2

\section{Resumen estadístico de la serie}

\begin{tabular}{|c|c|c|c|c|c|}
\hline Desviación & Varianza & $\begin{array}{c}\text { Número de } \\
\text { observaciones }\end{array}$ & Máximo & Mínimo & Promedio \\
\hline 1,49 & 2,22 & 499 & 42,6 & 3,5 & 22 \\
\hline
\end{tabular}

Fuente: empresa de servicios de programación y software.

Elaboración propia.

Con la información obtenida, se procede a realizar los cálculos de los límites:

Límite inferior: $L=z \sigma \sqrt{d}$, que al reemplazar los valores se obtiene:

$$
\begin{gathered}
L=(2.23 * 1.49) * \sqrt{4} \\
L=(3.32) * 2 \\
L=6.7
\end{gathered}
$$


Límite deseable: $Z^{*}=\sqrt[3]{\frac{3 F \sigma^{2}}{1 V}}+L$ una vez aplicada la fórmula se obtiene:

$$
\begin{gathered}
Z=\sqrt[3]{\frac{3 * 0.019865 * 2.22}{4 * 0.000028}}+6.7 \\
Z=\sqrt[3]{\frac{0.13}{0.000110674}}+6.7 \\
Z=\sqrt[3]{1,197.2205}+6.7 \\
Z=(10.61)+6.7 \\
Z=\mathbf{1 7 . 3}
\end{gathered}
$$

Límite superior: $H^{*}=3 Z *-2 L$, reemplazando los valores en la fórmula se obtiene:

$$
\begin{gathered}
H=(3 * 17.3)-(2 * 6.7) \\
H=(51.8)-(13.3) \\
\boldsymbol{H}=\mathbf{3 8 . 5}
\end{gathered}
$$

Una vez calculado los límites con el modelo MOV, se obtienen los siguientes valores para los diferentes niveles de riesgo (ver tabla 3):

Tabla 3

\section{Resultados modelo MOV de tesorería de la empresa}

\begin{tabular}{|c|c|c|c|}
\hline Conceptos & Límite inferior & Límite medio & Límite superior \\
\hline Saldo tesorería & 6,7 & 17,3 & 38,5 \\
\hline
\end{tabular}

Fuente: empresa de servicios de programación y software.

Elaboración propia.

Los límites calculados en base a información de 2016 y 2017 podrán ser utilizados a lo largo de 2018 para poder generar un correcto manejo operativo y abastecimiento sin alcanzar niveles críticos para la empresa.

En el gráfico 2 se puede observar cómo se comportó la serie real respecto a los límites calculados durante 2016 y 2017. El saldo de tesorería de la em- 
presa en este período osciló principalmente entre el límite superior y el límite medio, el cual es el nivel deseable. Sin embargo, se observa que se registraron períodos en los que la empresa presentó un manejo ineficiente de su saldo de tesorería, debido a que permitió que se alcancen niveles bajos de liquidez y, de igual manera, existieron momentos en los que el saldo estuvo por encima del límite superior, lo que significó un alto costo de oportunidad para la empresa.

Gráfico 2

\section{Límites para los niveles óptimos*}

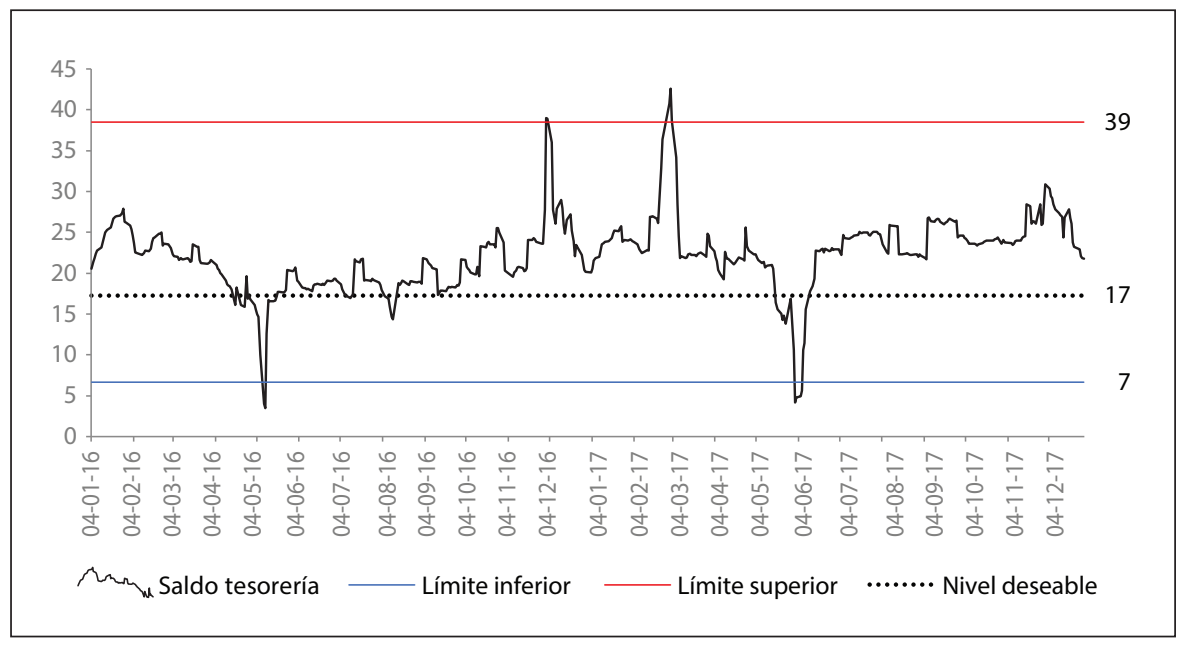

*En miles de dólares. Entre enero de 2016 y diciembre de 2017.

Fuente: empresa de servicios de programación y software.

Elaboración propia.

El comportamiento del saldo de tesorería de la empresa entre 2016 y 2017 no siempre se comportó dentro de los límites deseables evidenciando un manejo no necesariamente óptimo de su liquidez. Entonces, debido a que los límites son utilizados para establecer planes de contingencia de las empresas, en el gráfico 3, se observa el comportamiento de los saldos de tesorería de la empresa también para 2018, período en el cual la empresa hizo uso de los límites calculados por el modelo para su manejo de tesorería; de esta manera, se evidencia la capacidad que el modelo tiene para ser utilizado en tiempo real. 
Gráfico 3

\section{Límites para los niveles óptimos*}

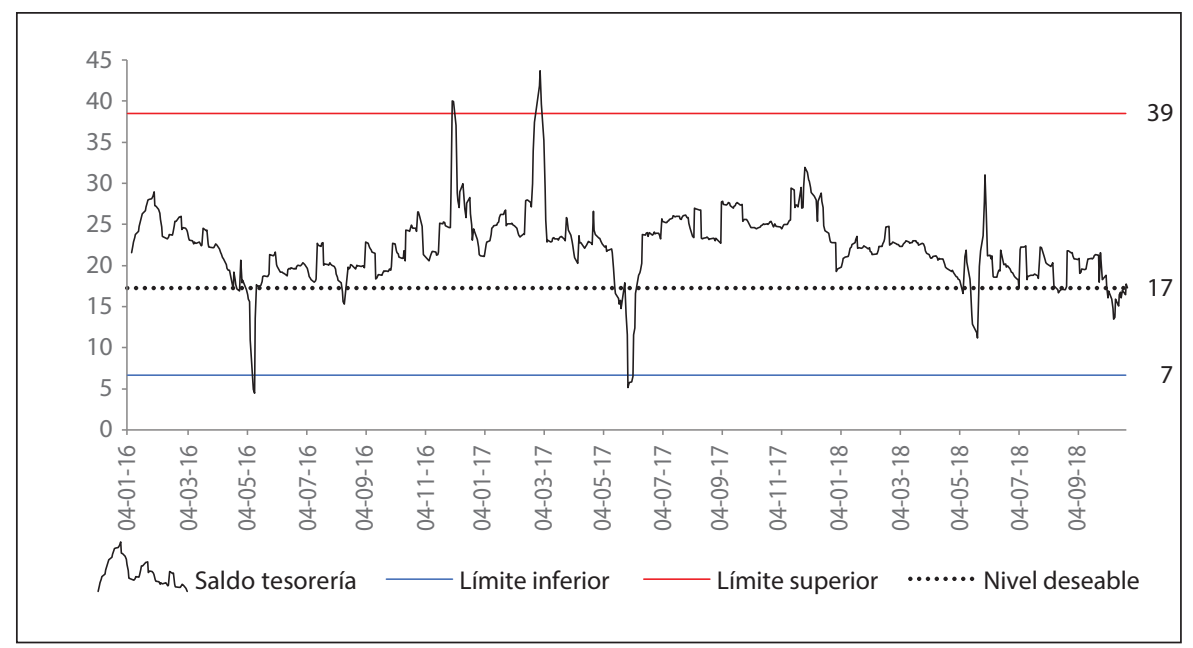

* En miles de dólares. Entre enero de 2016 y diciembre de 2018.

Fuente: empresa de servicios de programación y software.

Elaboración propia.

El comportamiento de la empresa en 2018 se encuentra dentro de los límites establecidos por el modelo, lo que indica un correcto manejo de sus niveles de efectivo; es decir, los límites planteados deben ser considerados como referenciales para el manejo de tesorería y efectivo. Por tanto, el saldo no debe ser exactamente igual a los límites calculados, sino que debe oscilar entre los límites extremos, orientado la gestión hacia el límite medio, respondiendo oportunamente a las necesidades de dinero en efectivo de la empresa.

Si el saldo se encuentra por encima del límite superior, esto supondría que la empresa se encuentra operando de manera ineficiente debido al costo de oportunidad de mantener parte de su liquidez en alternativas de inversión (adquisición de títulos valores, depósitos a plazo en el sistema financiero, entre otros) para acceder, así, a un rendimiento por sus colocaciones.

Es importante indicar que, si bien la modelización propuesta brinda una opción técnica adicional a los tomadores de decisiones en el manejo de tesorería, recogiendo el comportamiento estocástico en el flujo de caja, su limitación 
radica al no considerar fuentes tradicionales de financiamiento, como préstamos a corto plazo o sobregiros ocasionales, aplicados en la cotidianidad de las empresas al sobrepasar las necesidades de efectivo atípicas, que, si bien genera costos financieros, es una opción que se debe tomar en cuenta en situaciones de carácter excepcional cuando la necesidad de liquidez sobrepase las necesidades que son cubiertas con un manejo óptimo en condiciones normales.

\section{Conclusiones}

El modelo MOV nos permite optar por un nivel de saldo de efectivo objetivo menor en la tesorería de la empresa, si el costo de oportunidad (tasa de interés) es alto; con esto, las compañías maximizan su rentabilidad financiera, al optimizar el uso de los recursos monetarios disponibles.

La tesorería de la empresa debe plantearse como objetivo mantener un saldo de efectivo mayor si los costos de transacción que ameriten transformar la posición de efectivo a inversiones son altos, ya que cambiarse de posición entre efectivo e inversiones, le puede generar pérdidas financieras a la firma.

La incertidumbre en el manejo de la tesorería es un tema complejo, lo cual impide una administración eficiente del efectivo, puesto que cuando más alto es este elemento, mayor será la diferencia entre el saldo objetivo y el saldo de efectivo mínimo.

Una mayor incertidumbre también afecta al nivel máximo de tesorería, ya que se debe mantener en efectivo una cantidad superior para poder cubrir ciertas eventualidades, lo que hace que el saldo de efectivo promedio también sea alto.

Mientras más alta es la variabilidad de los flujos netos de efectivo, la posibilidad de que el saldo descienda por debajo del mínimo también será mayor, lo cual puede generar a la empresa incumplimientos con sus proveedores, clientes o usuarios en general.

La aplicación del modelo MOV en una empresa determinada permite mejorar la administración del efectivo, porque el mismo incorpora el efecto de la incertidumbre, medido a través de la variación de los flujos netos de efectivo. 
El modelo MOV adicionalmente presenta una particularidad respecto de los otros modelos, puesto que refuerza su liquidez a corto plazo, generando financiamiento desde el activo, sin tener que aumentar su pasivo financiero.

Los principios del modelo de tesorería de MOV pueden ser aplicados para establecer los niveles óptimos de efectivo que la empresa debe mantener para cubrir de manera eficiente los requerimientos constantes que pueda tener de dinero en efectivo, sin incurrir en costos innecesarios.

Los límites planteados deben ser considerados como referenciales para el manejo de tesorería y efectivo. Es decir, el saldo no debe ser exactamente igual a los límites calculados, sino que debe oscilar entre los límites extremos, orientado la gestión hacia el límite medio.

El modelo MOV de tesorería o de optimización del uso de efectivo, aplicado en este caso a un establecimiento de servicios, puede también ser utilizado en empresas comerciales, productivas, en entidades del sector financiero, bancos, cooperativas, inclusive en bancos centrales. ${ }^{5}$

Las contribuciones teóricas para la formulación del modelo permiten su utilización en cualquier tipo de economía, independientemente del esquema monetario, legislación tributaria y de otros elementos del entorno en que se desenvuelve la empresa. El modelo original de Miller y Orr data de varias décadas de aplicación empírica en el ámbito internacional y se mantiene vigente su marco teórico en los libros de textos de finanzas internacionales.

La aplicación del modelo MOV de efectivo se puede aplicar tanto a la tesorería nacional como internacional, considerando las propias particularidades de las empresas como los costos de transacción de liquidar activos y los costos de oportunidad asociados. Es posible que al optar por invertir en títulos valores por parte de la empresa, el hecho de generar una determinada utilidad le pueda generar costos tributarios o impuestos sobre las ganancias,

5. Para el caso de Colombia: "Los modelos documentados, a pesar de la fecha de su publicación, queda comprobado por los estudios académicos referidos que, gozan de vigencia y resultan oportunos, para que sean aplicados en empresas de Latinoamérica dado que la mayoría de las empresas son pequeñas o medianas y estos métodos son sencillos que no analizan economías muy complejas por lo que son de fácil adaptabilidad" (Castellanos 2019, 50). Vera (2017) plantea desde una perspectiva teórica la aplicación del modelo de demanda por dinero para empresas de Miller y Orr en la tesorería del banco central, a partir de lo cual el Banco Central del Ecuador utiliza como un instrumento adicional para el análisis de los flujos de liquidez. 
que debían ser considerados en los resultados netos; en efecto, la magnitud del efecto fiscal sobre las utilidades dependerá de la legislación tributaria de cada país, del tamaño y tipo de empresa que opte por aplicar este modelo, y de la dimensión de los flujos de liquidez que se canalicen a inversiones financieras, que al final tienen el propósito de optimar la tesorería de las firmas.

Los planteamientos teóricos que se establecen en el modelo se realizan a partir de aportes que datan de algunos años atrás como también de contribuciones recientes, lo que le da la robustez necesaria al tener una vigencia permanente en el tiempo, tanto en la literatura económica como en las aplicaciones empíricas.

\section{Referencias}

Aznar, Antonio, y Eduardo Pozo. 1998. "Demanda de dinero y tipos de interés: un estudio teórico". Revista Española de Economía 15 (1): 123-145. https://bit.ly/3bOb8un.

Baumol, William. 1952. "The Transactions Demand for Cash: An Inventory Theoretic pproach”. Quarterly Journal of Economics 66 (4): 545-556. doi:10.2307/1882104.

Feige, Edgar, y Michael Parkin. 1971. "The Optimal Quantity of Money, Bonds, Commodity Inventories and Capital". America Economic Review 61 (3): 335-349. 10.2307/2553324.

Fisher, Irving. 1986. "Appreciation and Interest". American Economic Association 3 (11): 331-442.

Goldman, Steven. 1974. "Flexibility and the Demand for Money". Journal of Economic Theory 9 (2): 213-222. 10.1016/0022-0531(74)90067-2.

Guissarri, Adrián. 1986. La demanda de circulante y la informalidad en la Argentina, 19301983. Buenos Aires: Instituto Torcuato Di Tella.

Higson, Andrew, Shinozawad Yoshikatsu y Mark Tippett. 2010. "Organization Size and the Optimal Investmen in Cash". Journal of Management Mathematics 21 (1): 27-38. 10.1093/imaman/dpp015.

Higuerey, Ángel. 2007. Administración del efectivo. Bogotá: Universidad de los Andes.

Jovanovic, Boyan. 1982. "Inflation and Welfare in the Steady State". Journal of Political Economy 90 (3): 561-577. 10.1086/261074.

Keynes, John. 1936. The General Theory of Employment. Londres: McMillan.

Krajewski, Lee, Larry Ritzman y Manoj Malhotra. 2013. Administración de operaciones: procesos y cadena de suministro. Ciudad de México: Pearson Educación.

Mies, Verónica, y Raimundo Soto. 2000. "Demanda por dinero: teoría, evidencia, resultados". Revista Economía Chilena 3 (3): 5-32. https://core.ac.uk/download/pdf/6642216.pdf. 
Modelo de demanda de dinero para firmas: aspectos teóricos, metodología y resultados

Milbourne, Ross. 1983. "Optimal Money Holding Under Uncertainty”. International Economic Review 24 (3): 685-698. 10.2307/2648794.

Miller, Merton, y Daniel Orr D. 1968. "The Demand for Money by Firms: Extensions of Analytic Results". Journal of Finance 23 (5): 735-759. 10.1111/j.1540-6261.1968. tb00314.x.

Pigou, Arthur. 1917. "The Value of Money”. The Quarterly Journal of Economics 32 (1): 3865. 10.2307/1885078.

Ramos, Vicente. 2015. "La demanda de dinero". En Introducción a la Macroeconomía, editado por Segundo Vicente Ramos, 24-54. Universidad del País Vasco/Euskal Herriko Unibertsitatea. https://ocw.ehu.eus/course/view.php?id=342.

Reyes, Yolanda. 2012. "Modelo de Merton Miller y Daniel Orr". Valparaíso: Pontificia Universidad Católica de Valparaíso. Serie de Apuntes Docentes. Accedido 15 de febrero. https://bit.ly/32eGWoZ.

Romer, David. 1987. "The Monetary Transmission Mechanism in a General Equilibrium Version of the Baul-Tobin Model". Journal of Monetary Economics 20 (1): 105-122. 10.1016/0304-3932(87)90060-2.

Santomero, Anthony. 1974. "A Model of the Demand for Money by Households". The Journal of Finance 29 (1): 89-102. 10.1111/j.1540-6261.1974.tb00026.x.

Tapiero, Charles, y Dror Zuckerman. 1980. "A Note on the Optimal Control of a Cash Balance Problem". Journal of Banking and Finance 4 (4): 345-352. 10.1016/0378-4266(80)900138.

Tobin, James. 1956. "The Interest Elasticity of the Transactions Demand for Cash". Review of Economics and Statistics 38 (3): 241-247. 10.2307/1925776.

Vera, Wilson. 2004. Reservas internacionales: nivel óptimo para el Ecuador. Quito: Escuela Politécnica Nacional.

---. 2017. Determinación de niveles óptimos de las reservas internacionales: una perspectiva teórica. Quito: Banco Central del Ecuador. Accedido 15 de febrero de 2020. https://contenido.bce.fin.ec/documentos/PublicacionesNotas/Catalogo/NotasTecnicas/nota80.pdf. 